\title{
Image Registration in Low Resolution Visual Sensor Networks
}

\author{
Harini Balasubramanian \\ hxbalasubramanian@wichita.edu \\ Praveen Kumar Mitikiri \\ pxmitikiri@wichita.edu \\ Kamesh R. Namuduri \\ kamesh.namuduri@wichita.edu \\ Department of Electrical and Computer Engineering \\ Wichita State University \\ Wichita, KS 67260.
}

\begin{abstract}
Image registration is of great interest in video surveillance because of its capability to combine images and generate a larger view of the area under observation, while retaining all the information in the images. This paper demonstrates a novel method for registering images obtained from low resolution visual sensor networks. It uses change detection as a tool for automatic identification of control points which form the basis for registering images.
\end{abstract}

\section{Introduction}

Surveillance over particular restricted regions in places such as museums, banks, etc is accomplished by the usage of wireless multi sensors with overlapping fields of view [1], [2]. In order to obtain a single image comprising information content from all the sensors, without any loss, the images have to be registered [3].

\section{Image Registration Algorithm}

\subsection{Experimental Setup}

The experimental set up consists of two visual sensors along with a host machine. Each sensor comprises of a Stargate sensor board along with the Phillips Logitech Pro 4000 web camera. The Stargate is a powerful single board computer with enhanced communication and sensor signal processing capabilities. It was developed by Intel and was licensed to Crossbow for production [4]. The Stargate board works on LINUX and also supports TinyOS based wireless sensor network technology. The web camera can take images with an image resolution 1.3 MPixels. The sensor board can communicate with the host machine in many different ways. The Stargate can be directly connected to a host machine through a serial port by giving the board and host machine a static IP address. This way the data from

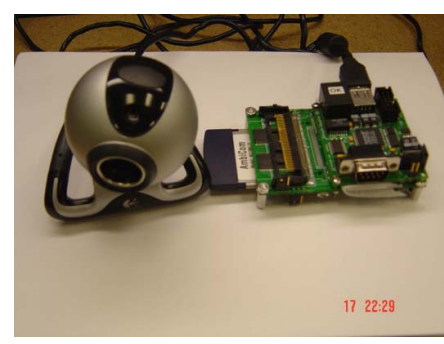

Figure 1. This figure shows the stargate sensor board and the web camera that form an image sensor node.

the sensor can be accessed securely by the host machine. The stargate board can also be connected to the internet using an RJ45 cable and it can be accessed by an internet enabled host machine using MOTEVIEW, which is a GUI used to view remote sensor images. In addition Stargate is equipped with an IEEE $802.11 a / b$ wireless card. For the experimental analysis, the sensor board was connected wirelessly to the host machine and the sensor board and the host machine were assigned static IP addresses. The host machine used for the experiments was a HP Pavilion laptop, with $2 G$ RAM. The images received by the host machine are registered using MATLAB. Figure 1 depicts a stargate sensor board and a web camera. 


\subsection{Image Acquisition and Change Detec- tion}

Let, $I_{11}, I_{12}$ be the images acquired by sensor $S_{1}$ at time instances $t_{1}$ and $t_{2}$ respectively. Let, $I_{21}$ and $I_{22}$ be the images collected by sensor $S_{2}$ at $t_{1}$ and $t_{2}$ time instances respectively. Let $D_{1}$ and $D_{2}$ be the difference images such that $D_{1}=\left|I_{11}-I_{12}\right|$ and $D_{2}=\left|I_{21}-I_{22}\right|$. A threshold $T h_{1}$, as in [4], is used to generate images $S D_{1}$ and $S D_{2}$ with only significant image difference pixels in $D_{1}$ and $D_{2}$. The changes in the overlapping region of fields of view of the two sensors, if any, are reflected in both the difference images.

\subsection{Control Point Selection}

$S D_{1}$ is called the base image and $S D_{2}$ is called the reference image. Each image is logically divided into blocks and if a block contains a significant pixel, then the number of other significant pixels in its neighborhood window is counted. If the count exceeds a threshold, then the point is marked as a potential control point. Once all the potential control points, if any, in a block are identified, the one that has the largest sum of intensity change in its neighborhood is selected as a control point. In this way, the control points for the two images are found. Let $C_{1 i}$ be the control points obtained from $S D_{1}$ and $C_{2 j}$ be the control points obtained from $S D_{2}$ where $i=1,2, \ldots n$ and $j=1,2, \ldots m$ where $n$ and $m$ are the total number of control points in $I_{12}$ and $I_{22}$ respectively. These control points may be located in the overlapping as well as non-overlapping fields of view since, change can occur in any part of the image. We need only corresponding control points in just the overlapping region of the two images to register them. This is done by finding the correlation between every possible pair of control points. Let $L C_{1 k}$ and $L C_{2 k}$ be the best correlated corresponding control points in the images, where $k=1,2,3 \ldots p$ and $p$ is the total number of corresponding points. These control points are used for transforming the reference image to the same spatial coordinates of the base image.

\subsection{Transformation and Registration}

In the proposed technique, considering the fact that the location and orientation of the sensors with respect to one another are unknown, affine transformation [5] is chosen. Affine transform is defined by a transformation matrix. From the transformation matrix, we can determinethe location of the sensors and orientation of one sensor with respect to the other. Once the images are transformed, the control points in the reference image are mapped into the transformed reference image to register them. This is achieved by applying the first two phases of the algorithm

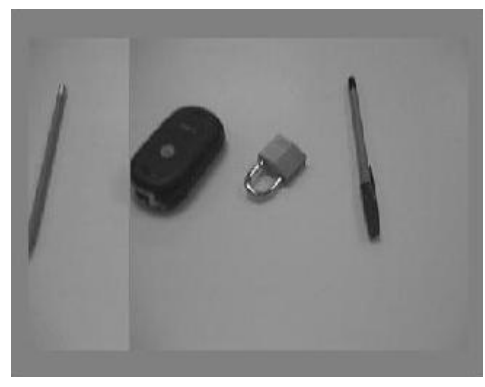

\begin{abstract}
Figure 2. The figure shows the final registered image formed by stitching the base image and the transformed reference image together.
\end{abstract}

to the transformed images from sensor $S_{2}$. Hereby the control points in the transformed reference image are obtained. Now, the transformed reference image and the base image are stitched together by identifying the location of the first pair of corresponding control points in the base image and the transformed reference image. The final registered image is as shown in Figure 2.

\section{Conclusion}

In this paper, we demonstrate an algorithm for automatic image registration in low resolution wireless visual sensor networks where precise and fine details may often be unavailable.

\section{References}

[1] L. G. Brown, "A survey of image registration techniques," ACM Computing Surveys, vol. 24, no. 4, December 1992.

[2] P. Kulkarni, D. Ganesan, and P. Shenoy, "The case for multi-tier camera sensor networks," Proceedings of the Internation Workshop on Network and Operationg Systems Support for Digital Audio and Video, pp. 141-146, June 13-14 2005.

[3] Z. Szlavik, T. Sziranyi, and L. Havasi, "Stochastic view registration of overlapping cameras based on arbitrary motion," IEEE Transactions on Image Processing, vol. 16, pp. 710-720, March 2007.

[4] S. Yelisetty and K. R. Namuduri, "Image change detection in wireless sensor networks," International Conference on Distributed Computing in Sensor Systems (DCoSS), June 2007.

[5] "2 $-d$ affine transformation," www.surv.ufl.edu. 